\title{
Type of the Paper: The Meaning Should Not Be Overlooked
}

\author{
Jitendra H. Mistry
}

Received: 11 July 2014 / Accepted: 22 July 2014 / Published online: 2 August 2014

(C) Association of Surgeons of India 2014

\section{Respected Sir,}

I read the article "Anatomic liver resection of segment 6, 7, and 8 by the method of selective occlusion of hepatic inflow" published in this journal [1]. The authors have presented a case of hepatocellular carcinoma involving segment 6,7 , and 8 with inadequate future liver remnant if right hepatectomy would have been performed, where they craftily performed anatomical resection of segment 6,7 , and 8 .

At the outset, I would like to mention that the intention of this letter is purely academic rather than criticizing someone. This paper was published under the section of "surgical techniques and innovations," so I started reading this article with expectation of technical details of this clever procedure. For anatomic segmental liver resection, the most important trick is to get the pedicle of a particular segment. In this particular case, to perform anatomical resection, we need to get pedicles at three different places to get the demarcation: (a) right Glissonean pedicle for getting demarcation between the right and left lobes of the liver, (b) right anterior or posterior pedicle to get demarcation between right anterior and right posterior sectors, (c) segment 5 or 8 pedicle to get demarcation between segment 5 and 8 . We can get right main and right anterior or posterior pedicles either extra or intrahepatic. It would be more helpful and would have carried out the meaning of this section of journal if the authors have mentioned in detail how they got these pedicles and which method was used. Even more important part of this procedure is to get and occlude the pedicle of segment 5 or 8 to get the demarcation between them. Authors have not clearly mentioned how they got demarcation between segment 5 and 8 , which is a critical technical part in this procedure for which the viewers are more interested.

As mentioned earlier, right main and right anterior or posterior pedicle can be approached extrahepatic or intrahepatic by hepatotomy. To get the pedicle of segment 5 and 8 , hepatotomy does require. In this case, after ligating the right posterior pedicle, initial parenchymal transaction along the demarcation line between the right anterior and posterior sector would help to follow the right anterior pedicle. Further, following the right anterior pedicle would identify the segment 5 and 8 division, and ligating segment 8 pedicle would help to get demarcation between segment 5 and 8 .

I conclude by saying that the authors had performed and described very important and interesting procedure, but providing the deeper detail of the technical part in their article would have better justified this section of the journal.

\section{Reference}

1. Jia C, Wang H, Chen Y, Fu Y (2014) Anatomic liver resection of segment 6,7 , and 8 by the method of selective occlusion of hepatic inflow. Ind J Surg 76(2):159-61
J. H. Mistry $(\bowtie)$

Kaizen Hospital, an Institute of Gastroenterology and Research

Centre, 132 f. Ring Road, Helmet Circle, Memnagar,

Ahmedabad 380052, Gujarat, India

e-mail: jitlap@gmail.com 